\title{
Reconstruction of the Primordial Power Spectrum using Temperature and Polarisation Data from Multiple Experiments
}

\author{
Gavin Nicholson* and Carlo R. Contald \\ Theoretical Physics, Blackett Laboratory, Imperial College, Prince Consort Road, London, SW7 2BZ, U.K.
}

(Dated: November 23, 2018)

\begin{abstract}
We develop a method to reconstruct the primordial power spectrum, $P(k)$, using both temperature and polarisation data from the joint analysis of a number of Cosmic Microwave Background (CMB) observations. The method is an extension of the Richardson-Lucy algorithm, first applied in this context by Shafieloo \& Souradeep [1]. We show how the inclusion of polarisation measurements can decrease the uncertainty in the reconstructed power spectrum. In particular, the polarisation data can constrain oscillations in the spectrum more effectively than total intensity only measurements. We apply the estimator to a compilation of current CMB results. The reconstructed spectrum is consistent with the best-fit power spectrum although we find evidence for a 'dip' in the power on scales $k \approx 0.002 \mathrm{Mpc}^{-1}$. This feature appears to be associated with the WMAP power in the region $18 \leq \ell \leq 26$ which is consistently below best-fit models. We also forecast the reconstruction for a simulated, Planck-like [2] survey including sample variance limited polarisation data.
\end{abstract}

\section{INTRODUCTION}

With increasingly precise measurements being made of the CMB (Cosmic Microwave Background) or LSS (Large Scale Structure) it becomes progressively more important to determine how new observations could yield the greatest insight into processes occurring in the early universe. One such observable is the primordial power spectrum of curvature perturbations $\Phi(\vec{k})$,

$$
P(k) \equiv \frac{k^{3}}{2 \pi^{2}} \delta^{3}\left(\vec{k}-\overrightarrow{k^{\prime}}\right)\left\langle\Phi(\vec{k}) \Phi^{*}\left(\overrightarrow{k^{\prime}}\right)\right\rangle,
$$

where $k$ is the wavenumber.

A generic prediction of the simplest inflationary models is that the density perturbations should be adiabatic and near scale invariant. In these models the spectrum takes the form of a power law $\propto k^{n_{s}-1}$. Current limits on this parametrisation place the spectral index $n_{s} \approx 0.963$ [3]. More complex inflationary models such as those with features on the potential 4, 5, 6, 7, 8, 9, 10], a small number of $e$-folds [11, 12, 13], or other exotic inflationary models [14, 15, 16, 17, 18] can modify $P(k)$ in a manner not compatible with the simple power law description. There have been many parametric searches for the features produced by these models 11, 19, 20, 21, 22, 23, 24, 25, 26, 27, 28], although none have proved conclusive. On the other hand, there have been tantalising hints of anomalous features in the data, for example after the first year WMAP results were released, there were strong indications of a cut-off in $P(k)$ on large scales. With subsequent data releases the significance of this feature has been reduced, although future observations of the polarisation of the CMB may provide more conclusive evidence [13].

\footnotetext{
*Electronic address: gavin.nicholson05@imperial.ac.uk

${ }^{\dagger}$ Electronic address: c.contaldi@imperial.ac.uk
}

A more thorough search for features in $P(k)$ would be one in which the theoretical model is independent from the reconstruction. Methods such as the RichardsonLucy deconvolution [1, 29, 30], cosmic inversion 31, 32, 33, 34, 35], and other non-parametric approaches [19, 36, 37, 38, 39, 40, 41, 42, 43] all attempt to overcome theoretical bias. With current computing power these techniques are generally limited to recovering the spectrum for one set of assumed cosmological parameters. This allows one to use a fiducial CMB photon transfer function to integrate the primordial curvature perturbation into today's photon distribution perturbation. However this assumption hides a significant degeneracy between features in the primordial power spectrum and the physical parameters which determine the height and position of acoustic peaks in the CMB when using only total intensity data in the reconstruction process [44]. As such it is not clear what the significance of any features found in the reconstructed $P(k)$ should be. Adding polarisation information into the reconstruction or inversion will significantly reduce this degeneracy since the response of polarisation CMB spectra is phase-shifted with respect to the total intensity response.

The total intensity ( $T$ modes) of the CMB have been measured accurately by several instruments $3,45,46,47$, 48] to arcminute scales. Measurements in the gradient (E-mode) and curl like ( $B$-mode) polarisation components, and their correlation with the total intensity $T E$, and $T B$, lag behind due to their lower amplitude. However a number of experiments are now measuring $E$-mode polarisation with increasing signal-to-noise, starting with the first detection of $E E$ spectrum [49] and subsequent measurements $3,446,48,50,51]$. B-mode polarisation has yet to be measured. Primordial tensor fluctuations may have an impact on the reconstruction of $P(k)$ on large scales. However, $B$-modes have not been detected yet and we neglect their contribution in this work.

We show in this paper that when information contained in both total intensity and polarisation radiation transfer functions is used in the reconstruction of the 
primordial power spectrum tighter constraints can be obtained. The paper is organised as follows; in section III we introduce the extension of the Richardson-Lucy algorithm used to estimate $P(k)$. In Section $\amalg I \mathrm{~A}$ we test the algorithm using simple forecasts of CMB data. Our tests include template input models with radically broken scale invariance. We explore current limits on $P(k)$ in section $\Pi I \mathrm{~B}$ and conclude in section IV]

\section{AN EXTENDED RICHARDSON-LUCY $P_{k}$ ESTIMATOR}

Direct primordial power spectrum reconstruction requires the inversion of the following relations

$$
C_{\ell}^{X Y}=\int_{-\infty}^{\infty} \frac{\mathrm{dk}}{k} \Delta_{\ell}^{X}(k) \Delta_{\ell}^{Y}(k) P(k),
$$

where $X$ and $Y$ represent $T, E$, or $B$-type anisotropies, $C_{\ell}^{X Y}$ are the angular power spectra for the $X Y$ combination and the $\Delta_{\ell}^{X}(k)$ are the photon perturbation transfer functions. The transfer functions are obtained by integrating the full Einstein-Boltzmann system of differential equations. These describe the evolution of perturbations in the photon distribution functions in the presence of gravity and other sources of stress-energy. The functions determine all of the structure in the anisotropy spectra which arises after the initial conditions are set. Most notably the $C_{\ell}^{X Y}$ contain distinct peaks due to the acoustic oscillation of the tightly coupled photon-baryon fluid in gravitational potential wells at the time of last scattering. The aim of any inversion method is to distinguish such features from any structure in the initial perturbation spectrum.

For a finite sampling of the wavenumber space $k$ Eq. (2) can be recast as an operator acting on the primordial spectrum $P_{k}$

$$
C_{\ell}=\sum_{k} F_{\ell k} P_{k}
$$

with operator

$$
F_{\ell k}^{X Y}=\Delta \ln k \Delta_{\ell k}^{X} \Delta_{\ell k}^{Y},
$$

where $\Delta \ln k$ are the logarithmic $k$ intervals for the chosen sampling.

A solution for $P_{k}$ cannot be obtained from a direct inversion of the $F_{\ell k}^{X Y}$ as it is numerically singular. This is due to the high level of degeneracy in the transfer functions relating the power at any wavenumber $k$ to angular multipoles $\ell$ although the system can be inverted by binning or smoothing appropriately to reduce the degeneracy [52].

An alternative approach is the iterative inversion employing the Richardson-Lucy (RL) method [53, 54] for image reconstruction. The RL method has been widely used in enhancing telescope images [55, 56, 57] and it can be shown that the RL method converges to the maximum likelihood estimator in the case of a Poisson distributed signal [58]. In the following we outline the use of the RL estimator in reconstructing the primordial power spectrum as introduced by Shafieloo et al. [1] and extend it to include properly weighted contributions from polarisation measurements.

Consider the case where the original source plane is the isotropic, primordial Fourier space spanned by the wavenumber $k$ and the convolved image plane is the space of angular multipoles $\ell$. In this case the convolution filter is $F_{\ell k}$ which relates modes the source power $P_{k}$ to the image power $C_{\ell}$.

The RL method provides an iterative solution to (3) for $P_{k}$, given an observed $C_{\ell}^{\text {obs }}$ with

$$
P_{k}^{i+1}=P_{k}^{i} \sum_{\ell=\ell_{\min }}^{\ell_{\max }} \tilde{F}_{\ell k} \frac{C_{\ell}^{\mathrm{obs}}}{C_{\ell}^{i}},
$$

where $C_{\ell}^{i}$ is the image obtained from the $i^{\text {th }}$ iteration $P_{k}^{i}$ and $\tilde{F}_{\ell k}=F_{\ell k} / \sum_{\ell} F_{\ell k}$ such that in the limit $C_{\ell}^{i} \rightarrow C_{\ell}^{\text {obs }}$ we have $\left(P_{k}^{i+1}-P_{k}^{i}\right) \rightarrow 0$. It is also convenient to recast (55) as change in the $P_{k}$ [1]

$$
P_{k}^{i+1}=P_{k}^{i}\left[1+\sum_{\ell=\ell_{\min }}^{\ell_{\max }} \tilde{F}_{\ell k} \frac{C_{\ell}^{\text {obs }}-C_{\ell}^{i}}{C_{\ell}^{i}}\right],
$$

such that the cut-offs in multipole space $\ell_{\min }$ and $\ell_{\max }$ are not propagated to the iterated solution through the broadening action of $\tilde{F}_{\ell k}$.

As it is (6) does not account for errors in the observed image as it applies uniform weighting to all $C_{\ell}^{\text {obs }}$. Since the RL estimator is not a well defined Maximum Likelihood estimator there is not a single choice of optimal weighting. Observational noise must therefore be included through an empirically chosen weighting function. We chose to add a weighting

$$
G_{k}=\frac{\sum_{\ell} \tilde{F}_{\ell k} C_{\ell}^{i}}{\sum_{\ell} \tilde{F}_{\ell k}\left[C_{\ell}^{i}+\sigma_{\ell}\right]},
$$

where $\sigma_{\ell}$ is the reported error in the $C_{\ell}^{\text {obs }}$. The weights $G_{k}$ have the properties that if $\sigma_{\ell} \ll C_{\ell}^{i}$ then $G_{k} \rightarrow 1$ and if $\sigma_{\ell} \gg C_{\ell}^{i}$ then $G_{k} \rightarrow 0$. The weighted estimator (6) now becomes

$$
P_{k}^{i+1}=P_{k}^{i}\left[1+G_{k} \sum_{\ell} \tilde{F}_{\ell k} \frac{C_{\ell}^{\mathrm{obs}}-C_{\ell}^{i}}{C_{\ell}^{i}}\right] .
$$

Most CMB experiments observe only part of the sky which leads to correlated $C_{\ell}$. The estimator does not account for the correlations as it includes only diagonal estimates of the uncertainty in $C_{\ell}^{\text {obs }}$. Indeed even fullsky experiments such as WMAP have correlated $C_{\ell}$ due to galaxy and source cuts. Experiments observing only 
small fractions of the sky usually report results in as a set of bandpowers $C_{b}=\sum_{\ell} W_{\ell}^{b} C_{\ell}$, where $W_{\ell}^{b}$ are the bandpower window functions. The bandpowers are less correlated than individual multipoles and allow us to apply the RL estimator to cut-sky experiments by calculating band filtered transfer function operators

$$
C_{b}=\sum_{\ell k} W_{\ell}^{b} F_{\ell k} P_{k} \equiv \sum_{k} F_{b k} P_{k} .
$$

Using this we can define a generalised iterative estimator with contributions from any number of bandpowers $b$ as

$$
P_{k}^{i+1}=P_{k}^{i}\left[1+G_{k} \sum_{b} \tilde{F}_{b k} \frac{C_{b}^{\mathrm{obs}}-C_{b}^{i}}{C_{\ell}^{i}}\right],
$$

where the $W_{\ell}^{b} \rightarrow \delta_{\ell \ell^{\prime}}$ in the full-sky limit (WMAP) and are the reported bandpower window functions for other experiments. In this work we will be using bandpowers from ACBAR [45], QUaD [46], BOOMERanG 47] and CBI [48] which increase the range of scales probed past WMAP's resolution limits.

A problem faced by the RL estimator when including polarisation measurements is that the polarisation bandpowers are correlated with the total intensity ones and cannot be included as further linear contributions to the sum in (10). Following the standard procedure for extending Maximum Likelihood estimators to polarised bandpowers we treat each multipole measurement as a $2 \times 2$ matrix of bandpowers with

$$
\mathbf{C}_{b}=\sum_{\ell}\left(\begin{array}{ll}
W_{\ell}^{b T T} C_{\ell}^{T T} & W_{\ell}^{b T E} C_{\ell}^{T E} \\
W_{\ell}^{b T E} C_{\ell}^{T E} & W_{\ell}^{b E E} C_{\ell}^{E E}
\end{array}\right),
$$

and similarly elevated the transfer function operator to a $2 \times 2$ matrix

$$
\mathbf{F}_{b k}=\sum_{\ell}\left(\begin{array}{ll}
W_{\ell}^{b T T} F_{\ell b}^{T T} & W_{\ell}^{b T E} F_{\ell b}^{T E} \\
W_{\ell}^{b T E} F_{\ell b}^{T E} & W_{\ell}^{b E E} F_{\ell b}^{E E}
\end{array}\right),
$$

and we have disregarded any $B$-mode contribution since current observational limits do not warrant its inclusion. We can now extend the RL estimator to include the full polarisation information as

$$
\begin{aligned}
P_{k}^{i+1}= & P_{k}^{i}(1+ \\
& \left.\operatorname{Tr}\left[\mathbf{G}_{k} \cdot \sum_{b} \tilde{\mathbf{F}}_{b k} \cdot\left(\mathbf{C}_{b}^{\text {obs }}-\mathbf{C}_{b}^{i}\right) \cdot\left(\mathbf{C}_{b}^{i}\right)^{-1}\right]\right),
\end{aligned}
$$

where $\mathbf{G}_{k}$ is obtained by modifying (7) to sum over banded quantities and $\tilde{\mathbf{F}}_{b k}$ is normalised so that the sum over its diagonal elements is unity.

We have implemented the estimator (13) to reconstruct $P_{k}$ over a discretely sampled grid in $k$ from a combination of CMB measurements listed above and for forecasted future data as described below. The full iteration typically takes $\mathcal{O}(10)$ minutes on a current desktop CPU. As a convergence criterion we iterate until the fractional change in the $P_{k}$ solution is less than 0.01 . Typically this takes $\mathcal{O}(100)$ iterations to achieve. We reconstruct $P_{k}$ over some $3000 k$ points between $6.9 \times 10^{-6} \mathrm{Mpc}^{-1}$ and $0.55 \mathrm{Mpc}^{-1}$. The sampling is initially logarithmic and switches to linear at $k=1.25 \times 10^{-3} \mathrm{Mpc}^{-1}$ following the typical setup in Einstein-Boltzmann solvers such as CAMB [59] but with higher resolution.

As shown in [1] the RL method requires a smoothing of the recovered $P_{k}$. We have found that applying a smoothing kernel at each iteration improves the convergence when using unbinned $C_{\ell}$ such as the WMAP results in the estimate. The smoothing helps to damp down large fluctuations at each iteration driven by the scatter in the observed data, due to either sample or noise variance. The behaviour is due to the estimator not being a well defined maximum likelihood one and thus not taking into account the full correlation structure of the data in its weighting. We have chosen a simple smoothing kernel for the applications reported in this work by taking a nearest neighbour running average over the $P_{k}$ between each iteration. Given our $k$ sampling this defines a smoothing size of $\Delta \log k \approx 0.04 \mathrm{Mpc}^{-1}$ at low $k$ and $\Delta k \approx 2 \times 10^{-4} \mathrm{Mpc}^{-1}$ at high $k$. Other smoothing kernels may lead to more efficient behaviour of the estimator such as faster convergence but would be more computationally demanding and would lead to a further loss of resolution in $k$ space.

Since the estimator requires $\mathcal{O}(10)$ minutes to converge it is not currently possible to employ it as part of a full Markov Chain Monte Carlo parameter search in place of the standard primordial spectrum parameter; amplitude $A_{s}$ and spectral tilt $n_{s}$. This may be possible in future if the algorithm is optimised and/or parallelised but in this work we chose to explore the $P_{k}$ "source" plane assuming a set of fixed fiducial transfer functions based on the bestfit parameters. The transfer functions are obtained from CAMB with parameters $\Omega_{b} h^{2}=0.0226, \Omega_{c} h^{2}=0.108$, $\theta=1.041$, and $\tau=0.076$.

To estimate the confidence limits around the sampled $P_{k}$ solution we Monte Carlo the iterative estimator by generating 1000 simulated sets of $C_{\ell}^{\text {obs }}$. For simplicity we assume Gaussian distributions for all the $C_{\ell}^{\text {obs }}$ since more accurate distributions are not easily reconstructed from some of the published data. We iterate to convergence on each of the simulated data sets and obtain a covariance matrix for the samples by averaging over the ensemble

$$
\sigma_{k k^{\prime}}^{2}=\frac{1}{N-1} \sum_{n=1}^{n=N}\left(P_{k}^{n}-\left\langle P_{k}\right\rangle\right)\left(P_{k^{\prime}}^{n}-\left\langle P_{k^{\prime}}\right\rangle\right)
$$

with $\left\langle P_{k}\right\rangle=\sum_{n=1}^{n=N} P_{k} / N$. We quote 1 and $2-\sigma$ errors by taking multiples of the square root of the diagonal elements of the $\sigma_{k k^{\prime}}^{2}$ matrix. 


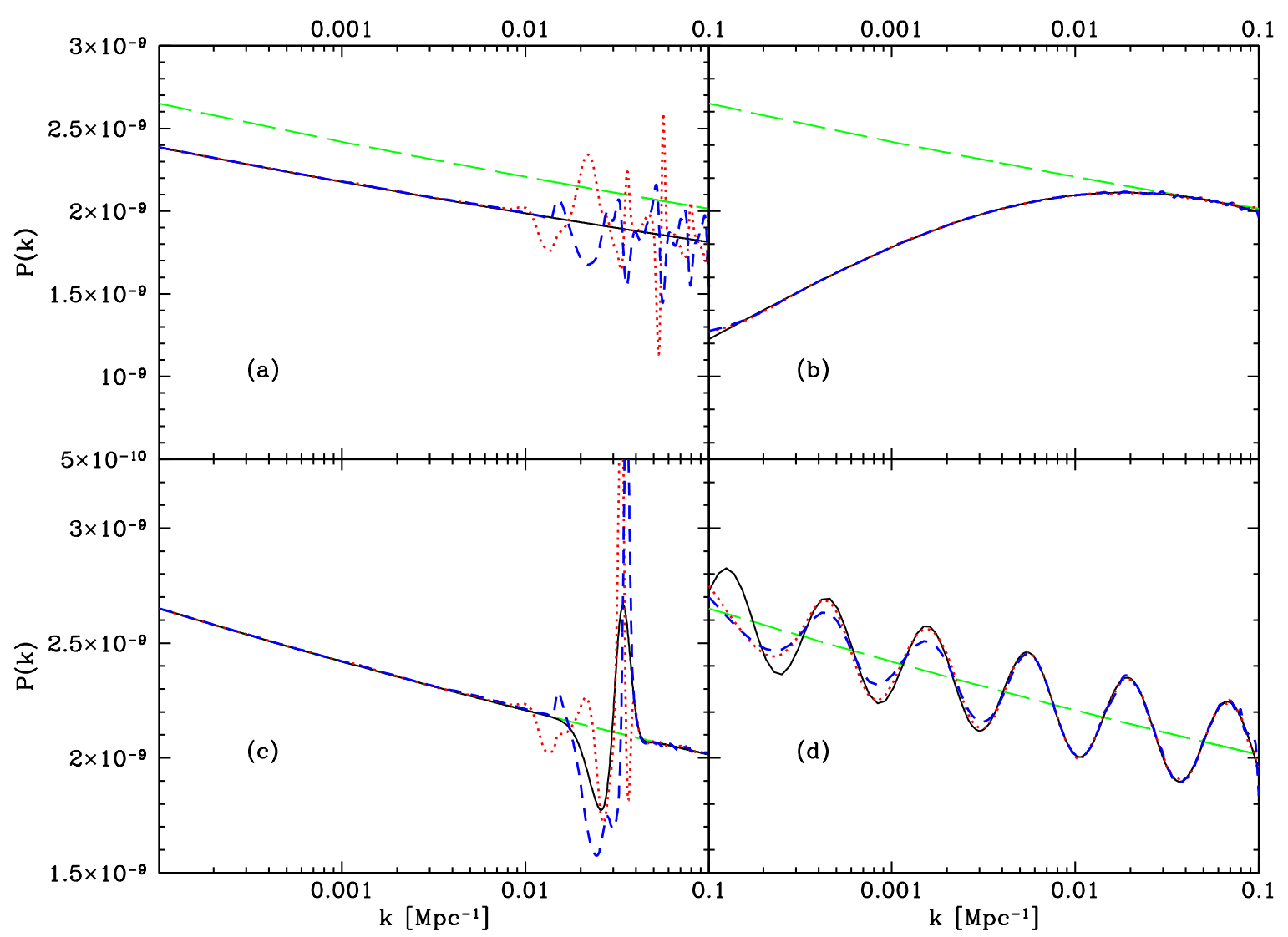

FIG. 1: The reconstruction of several test spectra. The test models used to generate the simulated $C_{\ell}$, shown in black (solid) curves, are (a) A 10\% decrease in power from the WMAP5 best fit amplitude, (b) the WMAP5 best fit model including running $d n_{s} / d \ln k=-0.037$, (c) a localised feature at around $k=0.02 \mathrm{Mpc}^{-1}$, and (d) a model with sinusoidal oscillations superimposed on the best fit power law spectrum. The green (solid) curves show the best fit spectrum used as initial guess in the iteration. The red (dotted) curves are the converged reconstructions using only total intensity data whereas the blue (dashed) curves use both total intensity and polarisation data. The $C_{\ell}$ forecasts assumed an experiment with similar properties to Planck.

\section{APPLICATION OF THE EXTENDED RL ESTIMATOR}

\section{A. Tests on forecasted CMB data}

We have tested the extended RL estimator on a number of input $P_{k}$ templates using forecasted data with similar experimental properties as the upcoming Planck satellite mission [2]. We assume a total of 12 detectors with NETs of $64 \mu K / \sqrt{s}$ observing $80 \%$ of the sky over 12 months with a resolution of 7 arcminutes FWHM. We calculate errors around our fiducial CMB best-fit models in both total intensity and polarisation spectra for this experimental setup and use these together with $C_{\ell}$ samples on the fiducial model to test the estimator's convergence. We consider multipoles of $\ell<1900$ for total intensity spectra and $\ell<1000$ for polarisation. We have not taken into account any residual error from foreground subtraction in our forecasts. Thus our forecast are on the optimistic side of the accuracy achievable, particularly in the polarisation where foreground removal will certainly have a significant impact on errors at $\ell<1000$.

We use four separate $P_{k}$ templates to generate the $C_{\ell}$ s. The first is a standard power law with $n_{s}=0.96$ but with a lower amplitude than the best-fit, the second is a running $n_{s}$ model with $d n_{s} / d \ln k=-0.037$, the third is a power law with a sharp, compensated feature at $k=$ $0.02 \mathrm{Mpc}^{-1}[4,7,8,10,14,15,16,17]$ and the fourth is a power law with superimposed sinusoidal oscillations $[5,69,18,60,61]$. The extended estimator is run on all four sets of $C_{\ell}$ forecasts and the resulting $P_{k}$ solution is compared to the input one. In Fig. 1 we show the results for runs including $C_{\ell}^{T T}$ only and runs including $C_{\ell}^{T T}$, $C_{\ell}^{T E}$, and $C_{\ell}^{E E}$.

Each run is started with a first guess $P_{k}$ (green/longdashed) which is the current best-fit power law spectrum. In all cases the structure in the input $P_{k}$ is reproduced to some degree over a large range in $k$. Beyond the $k$ range 

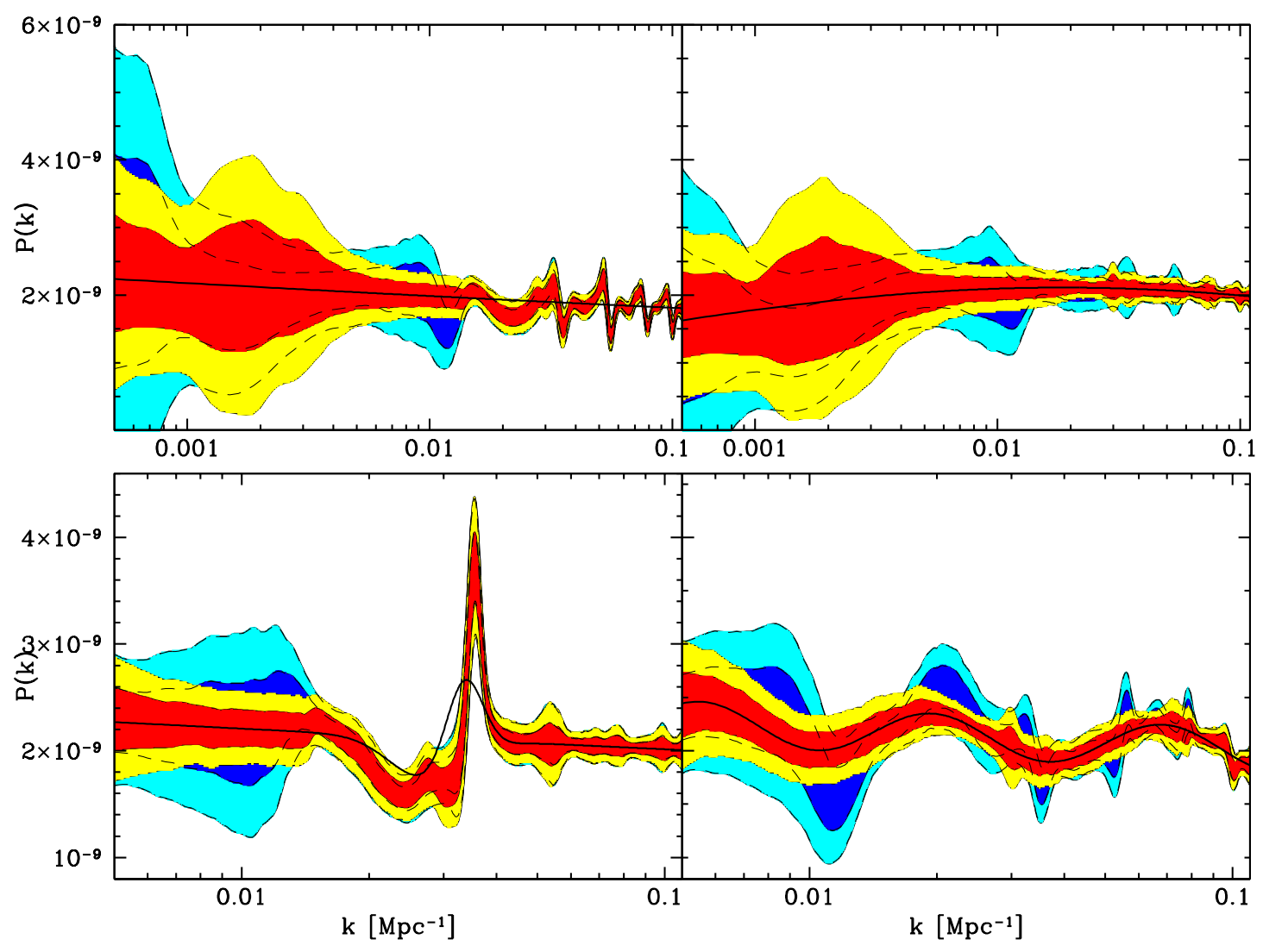

FIG. 2: Confidence regions around the reconstructed $P_{k}$ for the same test models shown in Fig. 1 The shaded areas indicate the 1 and $2-\sigma$ confidence regions obtained from 1000 Monte Carlo realisations of the observed $C_{\ell}$. The red (solid contours) shows the result for polarisation data included overlaid on the blue (dashed contours) showing the result for only TT included. The inclusion of polarisation has the largest impact in the case with superimposed oscillations.

shown in Fig. 1 there are significant departures from the correct solution. On the large scales $\left(k<0.0001 \mathrm{Mpc}^{-1}\right)$ this is due to the cut-off in the transfer functions due to the horizon scale. At small scales $\left(k>0.1 \mathrm{Mpc}^{-1}\right)$ the cut-off is due to the resolution limit of the forecasts. We find the method is particularly well suited for reconstructing long wavelength structure such as in the running and oscillating model. Although the sharp feature in the third model is present in the reconstructed spectrum, its amplitude is not recovered accurately. This example highlights the limitations of such methods in reconstructing high frequency features in the primordial spectrum. In the first model we also see high frequency features on small scales, this is due to the difference in amplitude between the initial guess and correct solution. A power law initial guess with closer starting amplitude to the correct value reduces this small scale noise. This could be easily obtained by carrying out a standard power law fit to the data before running the reconstruction estimate.

In Fig. 2 we show the same set of reconstructions but include 1 and $2-\sigma$ confidence regions obtained from the Monte Carlo covariance matrix. We have over sampled the $P_{k}$ and care should be taken in interpreting the significance of any feature as the samples are highly correlated. The plot includes a set of contours for the reconstruction using only total intensity data (blue/dashed) and including polarisation (red/solid). The range in $k$ has been modified to emphasise the regions where the best limits are obtained. The addition of polarisation data gives additional constraints on the smaller scales particularly in the oscillating model case. This is not surprising since total intensity and polarisation measurements are complementary in differentiating any structure in the primordial spectrum from acoustic oscillations imprinted on the CMB at last scattering. The polarisation data has little effect in improving the constraints on the spectrum with a compensated feature although a detection of the feature is evident.

\section{B. Current Limits}

In this section we use the extended RL estimator to reconstruct the primordial power spectrum using cur- 


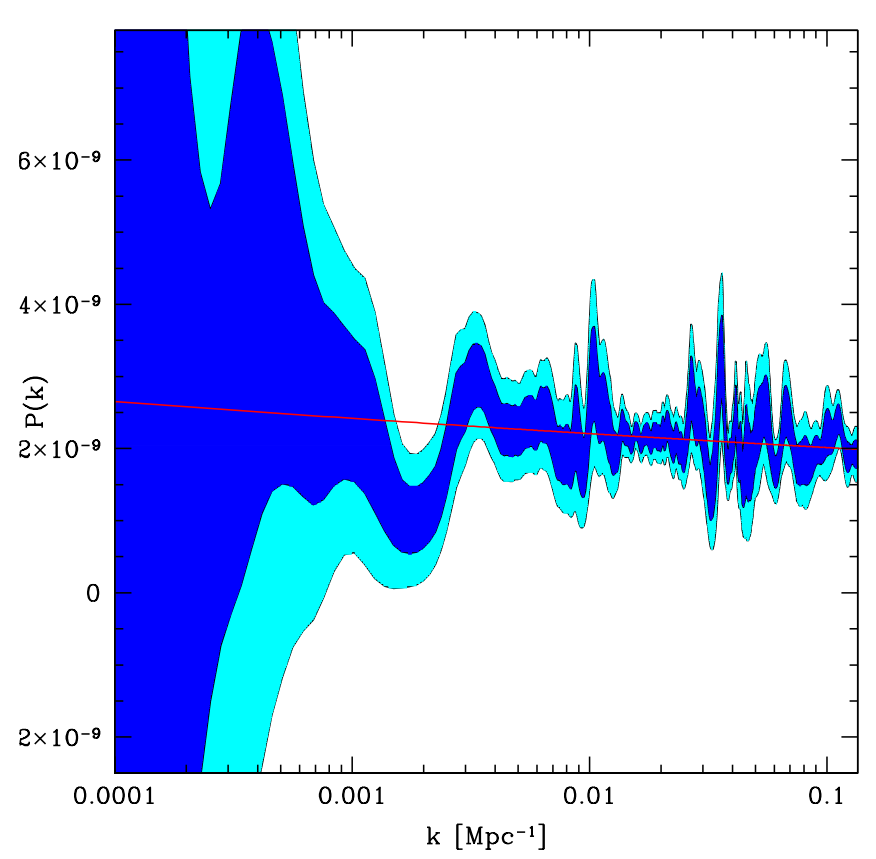

FIG. 3: Current limits from a combination of CMB data sets (WMAP, ACBAR, QUaD, BOOMERanG and CBI). There is some evidence of a dip in power at around $k \approx 0.002$ below the best fit power law model. Shaded regions are defined as in Fig. 2.

rently available data. Our combination of data includes the latest WMAP release [3] including $T T$ for $\ell<700$, the CBIpol results [48] including $T T$ band powers 2 to 12 and all polarisation band powers, the BOOMERanG 2003 [47] flight band powers, the QUaD [46] results, and the ACBAR 2008 [45] excluding the last two band powers due to the excess power. We have excluded the WMAP polarisation measurements as they are too noisy to be used in the reconstruction would require binning to reduce the scatter.

The overall effect of adding in the sub-orbital experiment is to extend the $k$ range of the reconstruction past $k \sim 0.07 \mathrm{Mpc}^{-1}$ where the WMAP noise becomes too large for the data to be included in the estimator. Given WMAP's resolution in multipole space, it strongly dominates the reconstruction on scales larger than $k \sim 0.07 \mathrm{Mpc}^{-1}$. In Fig. 3 we show the results of the reconstruction. There is no indication of a departure from a pure power law for the recovered spectrum except for a dip at $k \sim 0.002 \mathrm{Mpc}^{-1}$.

To determine the origin of the feature at $k \sim$ $0.002 \mathrm{Mpc}^{-1}$ we re-estimate the power spectrum with a cutout of the WMAP data in the range $18 \leq \ell \leq 26$ where the $C_{\ell}$ lie significantly below the best-fit models. Fig. 4 shows the result of for the reconstruction. No evidence for the feature remains when the $18 \leq \ell \leq 26$ WMAP data is removed from the estimate indicating that the dip is associated with the feature in the $C_{\ell}$.

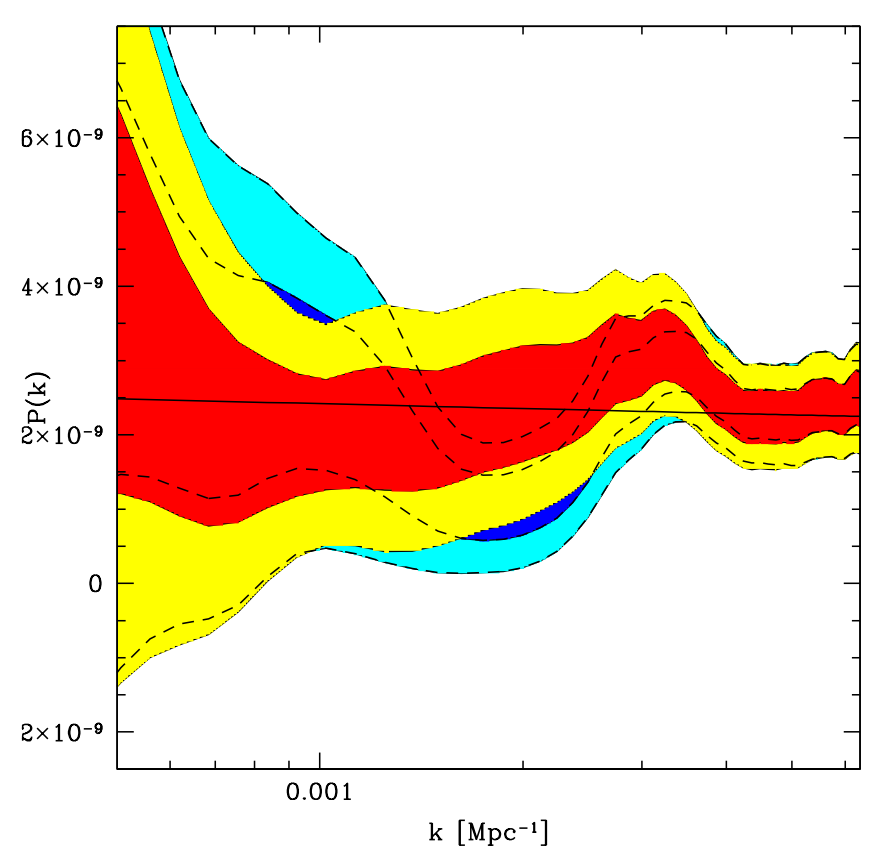

FIG. 4: As an indication of the origin of the dip at $k \approx 0.002$ $\mathrm{Mpc}^{-1}$ we remove the data between $\ell=18$ and $\ell=26$ and re-run the estimator. The red/yellow contours show the effect of the removal over the original estimate (blue/cyan).

\section{DISCUSSION}

The RL estimator provides a model independent method for reconstruction of the primordial power spectrum of perturbations $P_{k}$ from measurements of the CMB angular power spectrum $C_{\ell}$. We have extended the RL estimator to use on multiple data sets including properly weighted polarisation data and have used Monte Carlo realisations of the input $C_{\ell}$ measurements to estimate confidence limits around the reconstructed spectra.

We have applied the new estimator to current measurements. These include band powers from the QUaD experiment which provide the highest signal-to-noise measurements of $E E$ power so far. Including the polarisation information increases the constraints on the power spectrum reconstruction as it carries independent information which is complementary in phase to that of the total intensity. However current $T E$ and $E E$ measurements are still noise dominated and do not contribute significantly but future sample variance limited measurements will help to constrain any structure in the primordial spectrum as shown in our examples using forecasted data. An exception to this is the current WMAP polarisation data which may have some impact if it were binned to reduce noise scatter. We have not explored this option in this work as this would have required binning of the $T T$ data too with a resulting loss of resolution in $k$ space.

We have found that the addition of polarisation data is particularly helpful in constraining oscillatory structure 


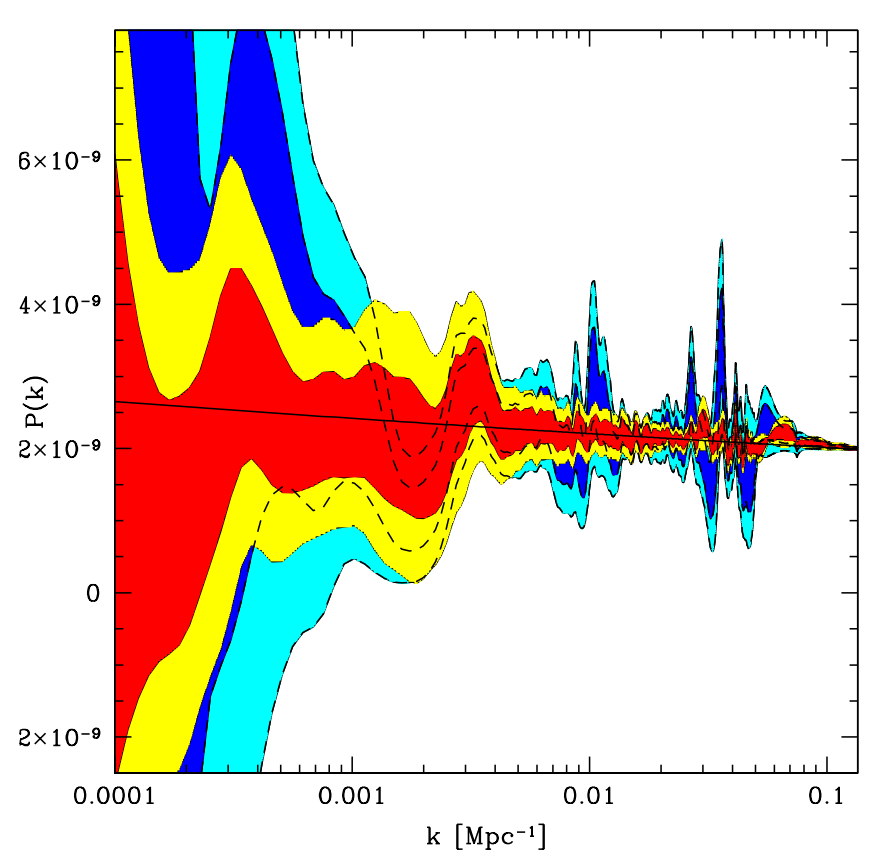

FIG. 5: We show the improvement that polarisation data can have on the estimation of the spectrum. The blue area is the uncertainty on $P_{k}$ given only the WMAP 5-year data. In the foreground we have combined this data with polarisation data from our Planck-like experiment and show that it significantly reduces the inherent error.

in $P_{k}$. Spectra with superimposed sinusoidal features have been considered in the literature and have been constrained using model dependent fits [9, 61, 62, 63].

Due to the empirical nature of the weighting used the estimator has a limited acceptance range in the signalto-noise of the band powers it is run on. Low signal-tonoise $C_{\ell}^{\text {obs }}$ or $C_{b}^{\text {obs }}$ cause the estimator to converge very slowly. Conversely if the data is weighted too strongly there is a loss of resolution and signal-to-noise in the reconstructed $P_{k}$. The best approach is to exclude the low signal-to-noise measurements from the data being used.

1 http://www.imperial.ac.uk/ict/services/teachingandresearchservices/highperformancecomputing
It is possible that other weighting schemes may be more efficient in allowing the whole range of $C_{\ell}$ measurements to be used. However, in its present form, the estimator will be most useful when all polarisations will be sample variance limited out to the same $\ell$. As an example of the improvement this will have over current estimates we add our forecasted $T E$ and $E E$ data to the current measurements and run the extended RL estimator. The result is shown in Fig. 5 which shows an overall improvement in the Monte Carlo confidence limits over the entire range in $k$ being reconstructed.

In this work we have not explored the degeneracy of the method with the physical parameters determining the structure of the CMB transfer functions. The current run-time to convergence for each reconstruction is too long to allow the tens of thousands of runs required to implement the method as part of a full Markov Chain Monte Carlo exploration of the entire parameter space. However with parallelisation and more efficient convergence it may become possible to do this in the near future.

There are further extensions of the RL estimator that could increase its effectiveness. The addition of other observables with different transfer functions such as galaxy redshift surveys or cosmic shear surveys will provide complementary information in the reconstruction. Our extension of the RL estimator is compatible with these other observables as they can be included as further independent data points with properly formatted and binned transfer functions. Such extensions would contribute key information in the reconstruction on scales where the presence of foregrounds in the CMB reduces its effectiveness.

\section{Acknowledgments}

This work was supported by a STFC studentship and used the Imperial College high performance computing service $^{1}$.
[1] A. Shafieloo and T. Souradeep, Phys. Rev. D70, 043523 (2004), astro-ph/0312174.

[2] Planck: The scientific programme (2006), astro$\mathrm{ph} / 0604069$.

[3] G. Hinshaw et al. (WMAP) (2008), astro-ph/0803.0732.

[4] J. A. Adams, B. Cresswell, and R. Easther, Phys. Rev. D64, 123514 (2001), astro-ph/0102236.

[5] X. Wang, B. Feng, M. Li, X.-L. Chen, and X. Zhang, Int. J. Mod. Phys. D14, 1347 (2005), astro-ph/0209242.

[6] P. Hunt and S. Sarkar, Phys. Rev. D70, 103518 (2004), astro-ph/0408138.

[7] M. Joy, V. Sahni, and A. A. Starobinsky, Phys. Rev. D77, 023514 (2008), 0711.1585.
[8] P. Hunt and S. Sarkar, Phys. Rev. D76, 123504 (2007), 0706.2443.

[9] C. Pahud, M. Kamionkowski, and A. R. Liddle (2008), 0807.0322.

[10] R. Lerner and J. McDonald, Phys. Rev. D79, 023511 (2009), 0811.1933.

[11] C. R. Contaldi, M. Peloso, L. Kofman, and A. Linde, JCAP 0307, 002 (2003), astro-ph/0303636.

[12] B. A. Powell and W. H. Kinney, Phys. Rev. D76, 063512 (2007), astro-ph/0612006.

[13] G. Nicholson and C. R. Contaldi, JCAP 0801, 002 (2008), astro-ph/0701783.

[14] J. Lesgourgues, Nucl. Phys. B582, 593 (2000), hep- 
$\mathrm{ph} / 9911447$.

[15] B. Feng and X. Zhang, Phys. Lett. B570, 145 (2003), astro-ph/0305020.

[16] G. J. Mathews, D. J. H. Chung, K. Ichiki, T. Kajino, and M. Orito, Phys. Rev. D70, 083505 (2004), astro$\mathrm{ph} / 0406046$.

[17] R. K. Jain, P. Chingangbam, J.-O. Gong, L. Sriramkumar, and T. Souradeep, JCAP 0901, 009 (2009), 0809.3915.

[18] A. E. Romano and M. Sasaki, Phys. Rev. D78, 103522 (2008), 0809.5142.

[19] S. L. Bridle, A. M. Lewis, J. Weller, and G. Efstathiou, Mon. Not. Roy. Astron. Soc. 342, L72 (2003), astro$\mathrm{ph} / 0302306$.

[20] D. Parkinson, S. Tsujikawa, B. A. Bassett, and L. Amendola, Phys. Rev. D71, 063524 (2005), astro-ph/0409071.

[21] R. Sinha and T. Souradeep, Phys. Rev. D74, 043518 (2006), astro-ph/0511808.

[22] C. Sealfon, L. Verde, and R. Jimenez, Phys. Rev. D72, 103520 (2005), astro-ph/0506707.

[23] P. Mukherjee and Y. Wang, JCAP 0512, 007 (2005), astro-ph/0502136.

[24] M. Bridges, A. N. Lasenby, and M. P. Hobson, Mon. Not. Roy. Astron. Soc. 369, 1123 (2006), astro-ph/0511573.

[25] M. Bridges, A. N. Lasenby, and M. P. Hobson (2006), astro-ph/0607404.

[26] L. Covi, J. Hamann, A. Melchiorri, A. Slosar, and I. Sorbera, Phys. Rev. D74, 083509 (2006), astro-ph/0606452.

[27] M. Joy, A. Shafieloo, V. Sahni, and A. A. Starobinsky (2008), 0807.3334.

[28] L. Verde and H. V. Peiris, JCAP 0807, 009 (2008), 0802.1219

[29] A. Shafieloo, T. Souradeep, P. Manimaran, P. K. Panigrahi, and R. Rangarajan, Phys. Rev. D75, 123502 (2007), astro-ph/0611352.

[30] A. Shafieloo and T. Souradeep, Phys. Rev. D78, 023511 (2008), 0709.1944.

[31] M. Matsumiya, M. Sasaki, and J. Yokoyama, Phys. Rev. D65, 083007 (2002), astro-ph/0111549.

[32] N. Kogo, M. Matsumiya, M. Sasaki, and J. Yokoyama, Astrophys. J. 607, 32 (2004), astro-ph/0309662.

[33] N. Kogo, M. Sasaki, and J. Yokoyama, Phys. Rev. D70, 103001 (2004), astro-ph/0409052.

[34] R. Nagata and J. Yokoyama, Phys. Rev. D78, 123002 (2008), 0809.4537.

[35] R. Nagata and J. Yokoyama, Phys. Rev. D79, 043010 (2009), 0812.4585.

[36] S. Hannestad, Phys. Rev. D63, 043009 (2001), astro- $\mathrm{ph} / 0009296$.

[37] Y. Wang and G. Mathews, Astrophys. J. 573, 1 (2002), astro-ph/0011351.

[38] P. Mukherjee and Y. Wang, Astrophys. J. 593, 38 (2003), astro-ph/0301058.

[39] P. Mukherjee and Y. Wang, Astrophys. J. 599, 1 (2003), astro-ph/0303211.

[40] S. Hannestad, JCAP 0404, 002 (2004), astro$\mathrm{ph} / 0311491$.

[41] D. Tocchini-Valentini, M. Douspis, and J. Silk, Mon. Not. Roy. Astron. Soc. 359, 31 (2005), astro-ph/0402583.

[42] S. M. Leach, Mon. Not. Roy. Astron. Soc. 372, 646 (2006), astro-ph/0506390.

[43] P. Paykari and A. H. Jaffe (2009), astro-ph/0902.4399.

[44] W. Hu and T. Okamoto, Phys. Rev. D69, 043004 (2004), astro-ph/0308049.

[45] C. L. Reichardt et al. (2008), astro-ph/0801.1491.

[46] C. Pryke et al. (QUaD) (2008), astro-ph/0805.1944.

[47] W. C. Jones et al., Astrophys. J. 647, 823 (2006), astro$\mathrm{ph} / 0507494$.

[48] T. J. Pearson et al., Astrophys. J. 591, 556 (2003), astroph/0205388.

[49] J. Kovac et al., Nature 420, 772 (2002), astro$\mathrm{ph} / 0209478$.

[50] T. E. Montroy et al., Astrophys. J. 647, 813 (2006), astro-ph/0507514.

[51] . C. Bischoff et al. (CAPMAP) (2008), 0802.0888.

[52] G. Nicholson (2009), in preparation.

[53] R. W. H., J. Opt. Soc. Am. 62, 55 (1972).

[54] L. B. Lucy, Astron. J. 79, 745 (1974).

[55] A. Jorissen, M. Mayor, and S. Udry (2001), astro$\mathrm{ph} / 0105301$.

[56] G. Surpi and R. D. Blandford, Astrophys. J. 584, 100 (2003), astro-ph/0111160.

[57] E. A. Helder and J. Vink (2008), 0806.3748.

[58] L. A. Shepp and Y. Vardi, Medical Imaging, IEEE Transactions on 1, 113 (1982), ISSN 0278-0062.

[59] A. Lewis, A. Challinor, and A. Lasenby, Astrophys. J. 538, 473 (2000), astro-ph/9911177.

[60] J. Martin and C. Ringeval, Phys. Rev. D69, 083515 (2004), astro-ph/0310382.

[61] J. Martin and C. Ringeval, JCAP 0501, 007 (2005), hepph/0405249.

[62] J. Hamann, L. Covi, A. Melchiorri, and A. Slosar, Phys. Rev. D76, 023503 (2007), astro-ph/0701380.

[63] J. Liu, H. Li, J. Xia, and X. Zhang (2009), 0901.2033. 